\title{
MANEJO DE INFECCIONES DE GARRAPATAS TRANSMITIDAS POR PERROS EN LA COMUNA DE PADRE LAS CASAS.
}

\section{TICKS INFECTIONS MANAGEMENT TRANSMITED BY DOGS IN PADRE LAS CASAS.}

\author{
Romina Álvarez. \\ Hospital Clínico Veterinario Comunal Ltda, Salvador 771-B, Providencia, Santiago. \\ Email: romina.alvarezrivas@gmail.com
}

Recibido: 02 de febrero de 2017; Aceptado: 15 de marzo de 2017.

La presencia de infecciones por garrapatas en perros implica un manejo sanitario externo López et al., (2013). Según un estudio de Dantas - Torres (2008), sugiere que uno de los grandes problemas en este punto es el mal uso de los acaricidas por parte de los propietarios. La aplicación de tratamientos antiparasitarios externos en los perros es un factor importante para el control de garrapatas, es por esto que dentro del plan de intervención.

Según Alcaíno et al., (1995) consideraba que la fumigación, existen estudios como los de García et al., (2007) que revelan las fases de vida libre de esta garrapata, mencionando que un gran número de ellas se encontraban dentro de las edificaciones. Según Dantas -Torres (2008), cuando se piensa en control de garrapatas se debe tomar en cuenta que solo el $5 \%$ de ellas se encuentran parasitando al perro y el $95 \%$ se encuentra en el ambiente. Se desprende del Reglamento para el control reproductivo de animales de compañía del Ministerio de Salud, que la tenencia responsable de mascotas implica tener obligaciones de parte del dueño enfocadas a proporcionarle alimento, albergue, buen trato, cuidados veterinarios indispensables para su bienestar y no someterlos a sufrimientos a lo largo de su vida.

Según los registros de la Municipalidad de Padre las Casas (2012), la encuesta CASEN 2000 reveló que existe un 19,2\% de la población en situación de pobreza, con un $8,8 \%$ de indigencia, por lo que se podría deducir que la atención veterinaria de las mascotas no se encuentra dentro de las prioridades de una familia. Según Medina (2011) la falta de responsabilidad de los propietarios puede radicar en la falta de educación, desinformación y situación socioeconómica deficiente, lo que puede 
conllevar a maltratos y abandonos por parte del dueño. Cabe agregar que en un estudio realizado en Argentina por Gervasoni et al., (2003), relaciona la baja atención veterinaria con los barrios más humildes, teniendo así mayores probabilidades de infestación por garrapatas en esos sectores.

Considerando que en Chile existe evidencia serológica de Ehrlichia sp (López et al., 2003) y Anaplasma sp en humanos (Abarca et al., 2008), se considera que el hecho de que existan viviendas con garrapatas intradomiciliarias es riesgoso para la salud de las personas. Se hace imperativo realizar más estudios sobre este tema para poder pesquisar futuros agentes que puedan afectar a la salud humana y animal del sector y la Región de La Araucanía.

En el año 2003 la OPS estimó que en América Latina existen 65 millones 130 mil perros y en Chile hay 2 millones 300 mil ejemplares. Guttler (2005), menciona que Latinoamérica presenta una relación de 1 perro cada 7,7 personas y en Chile existe una relación de 1 perro cada 6,8 personas. Según una publicación de WHO (1990), la relación de perro/ habitante en Estados Unidos y países de Europa era de 1:10 y 1:6 respectivamente, considerándose que estas son las recomendaciones que se deben seguir para evitar una sobrepoblación canina. Como una de las medidas de mitigación para la infestación por garrapatas en la comuna de Padre las Casas, se propone realizar un manejo de natalidad mediante esterilizaciones masivas, un censo canino y educación sobre la tenencia responsable de mascotas. Estas sugerencias ayudaran al control de garrapatas en la zona y además a tener una visión más clara frente a esta problemática a nivel comunal y regional.

Padre las Casas se caracteriza por tener un clima lluvioso especialmente en los meses de invierno. El mes más frío tiene una temperatura media entre $3^{\circ} \mathrm{C}$ y $18^{\circ} \mathrm{C}$ y la media del mes más cálido supera los $10^{\circ} \mathrm{C}$ (MPLC 2012). El mundo se encuentra en una situación de cambios climáticos y Chile no es la excepción. Las precipitaciones disminuirán de un $20 \%$ al $40 \%$ en todas las regiones del país y las sequias se harán más abundantes. Las temperaturas aumentaran de 2 a $4^{\circ} \mathrm{C}$ para fines del siglo XXI, por lo que se puede afirmar que el sur de Chile se verá cada año más afectado. Para Barcat (2006) una de las consecuencias que trae el calentamiento global es el aumento de las enfermedades transmitidas por garrapatas, lo que coincide con López \& Medina (2005) quien afirma que la temperatura es un factor crítico en que puede aumentar o disminuir la supervivencia de un vector, por lo que un leve cambio climático podría aumentar la población de garrapatas en una zona determinada. En un estudio de Cortes (2010), menciona que las garrapatas se pueden encontrar durante todo el año, pero en países donde existen las estaciones bien definidas se centran en los meses de 
verano y primavera por sus mayores temperaturas. Es recomendable realizar un plan de prevención en las épocas de verano y primavera, utilizando collares, baños sanitarios y pipetas, agregando a esta actividad un control domiciliario mediante fumigación del recinto. Desde la salud pública, sigue siendo una mejor opción utilizar productos que actúen por contacto y den muerte al parásito antes de morder al animal para evitar la transmisión de agentes infecciosos tanto al perro como al humano.

Es necesario que las organizaciones sociales y la comunidad se hagan cargo del problema parasitario en sus mascotas junto con un ente municipal. La educación de tenencia responsable, incluyendo ítems importantes como lo es el manejo sanitario de las mascotas, hábitos de vida y confinamiento, se hace primordial si se quiere controlar agentes que pueden llegar a afectar al ser humano.

\section{REFERENCIAS}

Abarca, K., López, J., González, P., Dabanch, J., Torres, M., Solari, V., Perret, C. 2008. Evidencia seroepidemiológica de exposición humana a Anaplasma $s p$ en Santiago, Chile. Revista Chilena de Infectología 25. 360.

Alcaíno, H., Gorman, T., Acosta, P., Fredes, F. 1995. Evaluación de cinco esquemas de control con cipermetrina del Rhipicephalus sanguineus en la Región Metropolitana de Chile. Archivos de Medicina Veterinaria, 27: 45-50.

Barcat, J. 2006. El calentamiento global, las garrapatas y la ehrlichiosis. Medicina 66: 489-491.

Cortés, J. 2010. Cambios en la distribución y abundancia de las garrapatas y su relación con el calentamiento global. Revista de Medicina Veterinaria 57: 33

Dantas- Torres, F. 2008. The brown dog tick, Rhipicephalus sanguineus (Latreille 1806) (Acari: Ixodidae): From taxonomy to control. Veterinary Parasitology, 152: $173-175$.

García, M., Moissant, E., Pérez, A., Quijada, J., Simoes, D., García, H. 2007. Comportamiento natural de las fases no parasíticas de Rhipicephalus sanguineus (latreille, 1806) (acari: Ixodidae) en un bioterio canino de Venezuela. Revista Científica Maracaibo, 17: 567-568.

Gervasoni, S., Guglielmone, A., Tarabla, H., Ruíz, M. 2003. Algunos factores de importancia en la infestación domiciliaria por garrapatas del grupo Rhipicephalus sanguineus (Latreille, 1806). Anuario 2003, UNL.

Guttler, V. 2005. Análisis de algunas características de la población canina relacionadas con mordeduras e hidatidosis humana en la provincia de Valdivia. Tesis de 
Grado. Universidad Austral de Chile, Facultad de Ciencias Veterinarias. Valdivia. Chile.

López, J., Peña, A., Pérez, R., Abarca, K. 2013. Tenencia de mascotas en pacientes inmunocomprometidos: actualización y consideraciones veterinarias y médicas. Revista Chilena de Infectología, 30: 52-62.

MPLC, Municipalidad Padre las Casas, Chile. 2012. Plan de desarrollo comunal comuna de Padre las Casas Región de la Araucanía.

Medina, C. 2011. La ética de la responsabilidad y el respeto a las mascotas -como formas de vida-, como solución al maltrato y abandono de las mismas. Tesis Maestría. Universidad del Bosque. Departamento de Bioética, Bogotá D.C.

WHO, World Health Organization/World Society for the Protection of Animals, Geneva. 1990. Guidelines for Dog Population Management. Pp. 9. 